\title{
Predicción de la calidad del aire multiescala con el modelo MONARCH en el Centro Nacional de Supercomputación
}

https://doi.org/10.31978/639-19-010-0.405

\author{
Oriol Jorba ${ }^{1}$ (oriol.jorba@bsc.es), \\ Sara Basart ${ }^{1}$, Jaime A. Benavides ${ }^{1}$, Dene Bowdalo ${ }^{1}$, Matthew L. Dawson ${ }^{1}$, \\ Enza Di Tomaso ${ }^{1}$, María Gonçalves ${ }^{1}$, Marc Guevara ${ }^{1}$, Martina Klose ${ }^{1}$, \\ Francesca Macchia ${ }^{1}$, Vincenzo Obiso ${ }^{1}$, Miriam Olid ${ }^{1}$, María Teresa Pay ${ }^{1}$, \\ Manuel Porquet ${ }^{1}$, Kim Serradell ${ }^{1}$, Carles Tena ${ }^{1}$ y Carlos Pérez García-Pando ${ }^{1}$
}

${ }^{1}$ Barcelona Supercomputing Center - Centro Nacional de Supercomputación / Departamento de Ciencias de la Tierra

\begin{abstract}
RESUMEN
El Departamento de Ciencias de la Tierra del Barcelona Supercomputing Center — Centro Nacional de Supercomputación (BSC-CNS) desarrolla el modelo de calidad del aire multiescala Multiscale Online Nonhydrostatic AtmospheRe CHemistry model (MONARCH) para la predicción de la calidad del aire a escala Global/Europea/Nacional/Local. Actualmente, el modelo proporciona la predicción operativa de transporte de polvo mineral en el centro especializado Barcelona Dust Forecast Center (https://dust.aemet.es/) y contribuye al centro regional Sand and Dust Storm Warning Advisory and Assessment System for Northern Africa-Middle East-Europe (http://sds-was.aemet.es/), ambos gestionados por AEMET y BSC-CNS. Complementariamente, MONARCH participa en la iniciativa International Cooperative for Aerosol Prediction (http://icap.atmos.und.edu/) como miembro de la predicción por conjuntos de aerosoles globales. El BSC-CNS mantiene desde hace años el sistema de predicción de la calidad del aire para España CALIOPE (http://www.bsc.es/caliopeles) y actualmente está implementando el modelo MONARCH como motor del mismo remplazando el sistema inicial basado en los modelos WRF y CMAQ. Asimismo MONARCH es uno de los nuevos candidatos que optan a integrarse en el sistema de predicción regional multimodelo para Europa (CAMS50).

En esta contribución se presentará una visión general del modelo MONARCH, los desarrollos recientes referentes a los procesos físico-químicos de la atmósfera, el acoplamiento con el nuevo modelo de emisiones multiescala HERMESv3, y su sistema de asimilación de datos basado en la metodología por conjuntos Local Ensemble Transform Kalman Filter. A continuación, se discutirán ejemplos de aplicación del mismo a distintas escalas espaciales: 1) predicción de aerosoles global a $50 \mathrm{~km}, 2$ ) predicción de ozono y material particulado en España a 4 km, y 3) predicción de óxidos de nitrógeno a escala urbana a 10 m. Finalmente, se mostrarán los trabajos en asimilación de datos de medidas satelitales de espesor óptico orientados a desarrollar análisis de aerosoles con especial énfasis en el polvo mineral.
\end{abstract}

PALABRAS CLAVE: predicción numérica; predicción de la calidad del aire; asimilación de datos. 
